\title{
Suture line recurrence in jejunal pouch replaced after total gastrectomy for gastric cancer
}

\author{
Kazuya Miyoshi ${ }^{1}$, Sadanori Fuchimoto ${ }^{1}$, Toshihide Ohsaki ${ }^{1}$, Tatsuhiko Sakata ${ }^{1}$, Isao Takeda ${ }^{1}$, \\ Kenji Takahashi ${ }^{1}$, Takaomi Ohkawa ${ }^{1}$, Kimiaki Tanaka ${ }^{1}$, Tomoko Matsumoto ${ }^{1}$, Norihisa Takakura ${ }^{2}$, \\ and Макото Мотог ${ }^{3}$ \\ ${ }^{1}$ Department of Surgery, Fukuyama National Hospital, 4-14-17 Okinogamicho, Fukuyama 720-0825, Japan \\ ${ }^{2}$ First Department of Surgery, Okayama University Medical School, 2-5-1, Shikatacho, Okayama 700-8558, Japan \\ ${ }^{3}$ Department of Pathology, Medical Checkup Center of Fukuyama Medical Association, 2-11-25 Miyoshichominami, \\ Fukuyama 720-0032, Japan
}

\begin{abstract}
:
We report a case of suture line recurrence in a jejunal pouch, diagnosed 4 months after total gastrectomy for advanced gastric cancer. The jejunal pouch was made with a linear stapler, without intraluminal irrigation being carried out before anastomosis, and was replaced in an interposition fashion. We propose that the recurrence was caused by the implantation of exfoliated cancer cells in either the intraluminal mucus or on a contaminated stapling device.
\end{abstract}

Key words: suture line recurrence, linear stapler, jejunal pouch, gastric cancer

\section{Introduction}

Suture line recurrence has been reported to develop sometimes after operations for colorectal cancer in which stapling devices are used. Implantation of exfoliated cancer cells during the performance of anastomosis has been considered a possible cause. Intraluminal irrigation with cytotoxic agents before anastomosis decreases the rate of suture line recurrence. There are few reports of suture line recurrence of gastric cancer, however, and similar preventive measures are not routinely performed. We report a case of suture line recurrence in a jejunal pouch replaced after total gastrectomy for advanced gastric cancer.

\section{Case report}

A 74-year-old man underwent total gastrectomy, extended lymph node dissection (D2), distal pancreatec-

Offprint requests to: K. Miyoshi

Received for publication on April 19, 1999; accepted on Sept. 7, 1999 tomy, and splenectomy because of gastric cancer, on September 13, 1995. The lesion was a macroscopic type 3 tumor $(5.0 \times 4.5 \times 1.0 \mathrm{~cm}$ in size $)$ in the posterior wall of the upper and middle third of the stomach (Fig. 1). The tumor consisted of well differentiated tubular adenocarcinoma (tub 1) and poorly differentiated adenocarcinoma, solid type (por 1) with scanty stroma (med) (Fig. 2). It had infiltrated into surrounding tissue, in part (infiltration [INF] $\beta$ ) associated with moderate lymphatic invasion (ly2) and no venous invasion (v0). The cancer had invaded the subserosa (pT2) and involved a lymph node along the lesser curvature (pN1). The final stage grouping was stage II and no residual disease with high probability of cure (resection A) [1].

Reconstruction was carried out in an interposition fashion with a jejunal pouch (Fig. 3). To construct the jejunal pouch, a plicated jejunal loop, measuring $12 \mathrm{~cm}$ in length, was brought up posteriorly to the transverse colon. A linear stapler (Proximate Linear Cutter 55; Ethicon Endo-Surgery, Cincinnati, OH, USA) was introduced at the mid portion of the jejunal loop, and a side-to-side anastomosis was performed at the antimesenteric borders of the bowel. An end-to-end jejunoduodenostomy approximately $9 \mathrm{~cm}$ below the pouch was created, using a circular stapler (Premium Plus CEEA 25; US Surgical, Norwalk, CT, USA). After end-to-side esophagojejunostomy was performed with a CEEA device, the center hole of the jejunal pouch was closed transversely in two layers. Intestinal continuity was re-established with an end-to-end jejunojejunostomy $20 \mathrm{~cm}$ distal to Treitz's ligament. No irrigation of the jejunum with a cytotoxic agent was performed before the creation of the anastomosis.

The patient made an uneventful recovery, but, 4 months after the operation, he complained of epigastric discomfort. Computed tomography revealed an intraluminal tumor in the jejunal pouch, without any other 


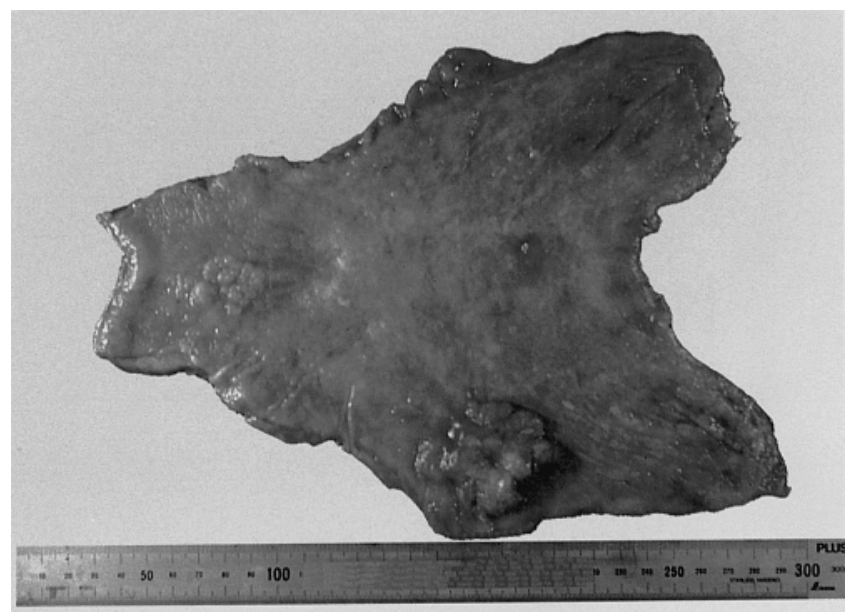

Fig. 1. The lesion was a macroscopic type 3 tumor in the posterior wall of the upper and middle third of the stomach

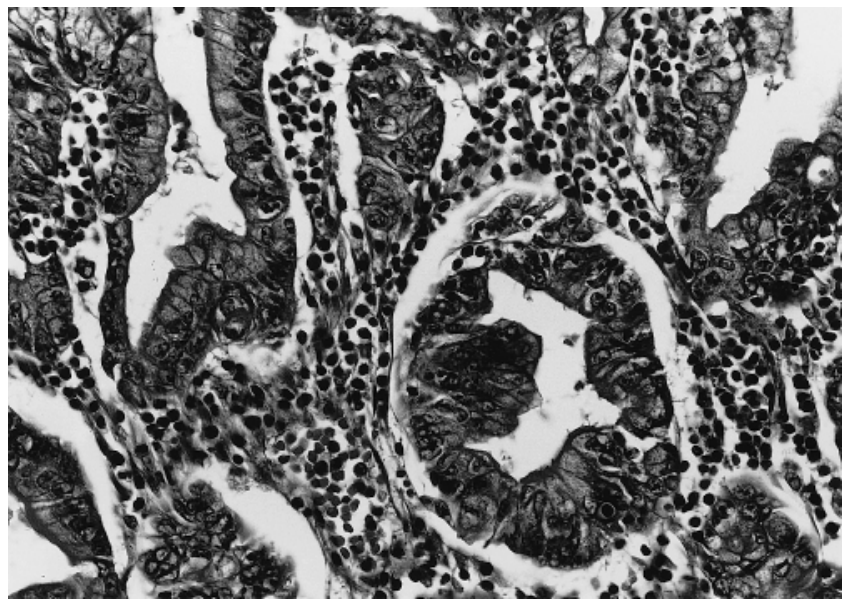

Fig. 2. The tumor consisted of well differentiated tubular adenocarcinoma (tub 1) and poorly differentiated adenocarcinoma, solid type (por 1) with scanty stroma (med). H\&E, $\times 80$

recurrent lesion (Fig. 4), and endoscopy showed four elevated, broad-based tumors in the jejunal pouch, along the suture line created by the linear stapler (Fig. 5). Well differentiated tubular adenocarcinoma (tub 1) was revealed in the biopsy specimens (Fig. 6). As the histological features of the new lesion were similar to those of the resected gastric cancer and there had been no tumor in the jejunum at the operation, it was reasonable to assume that the new lesion was a local recurrence of the gastric cancer resected 4 months earlier.

The patient refused salvage surgery and died on September 30, 1998 because of progression of the recurrent cancer. Autopsy was not performed.

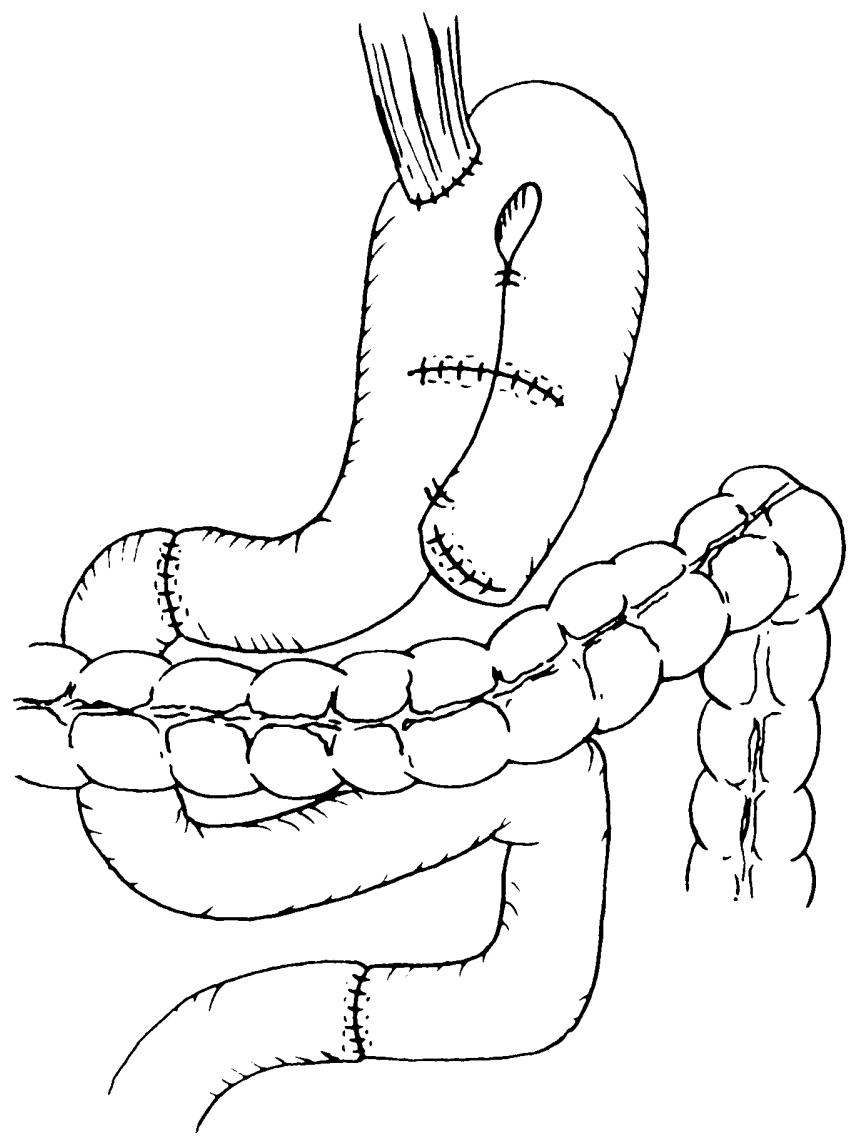

Fig. 3. Reconstruction was carried out in an interposition fashion with a jejunal pouch

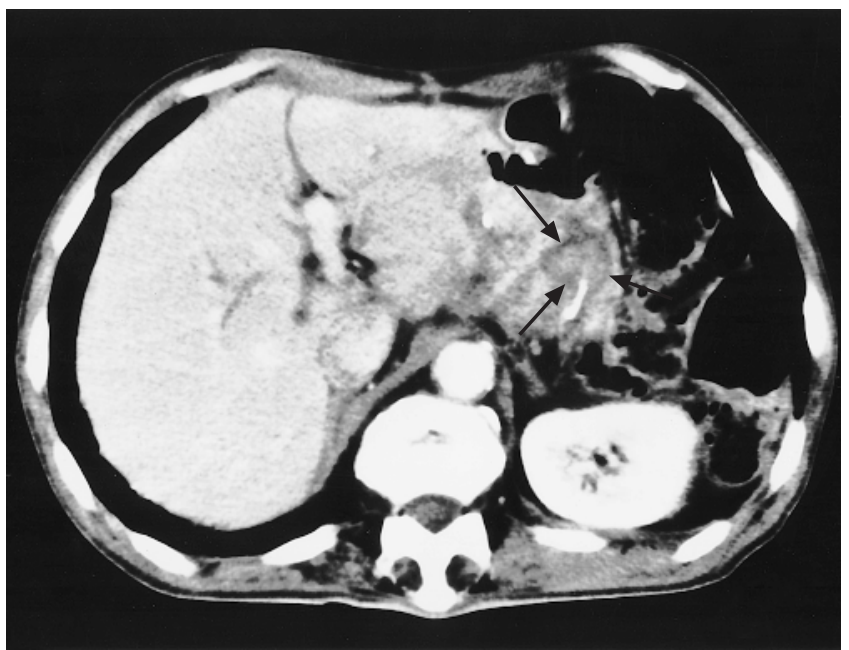

Fig. 4. Computed tomography revealed intraluminal tumor in the jejunal pouch (arrows) without any other recurrent lesion 


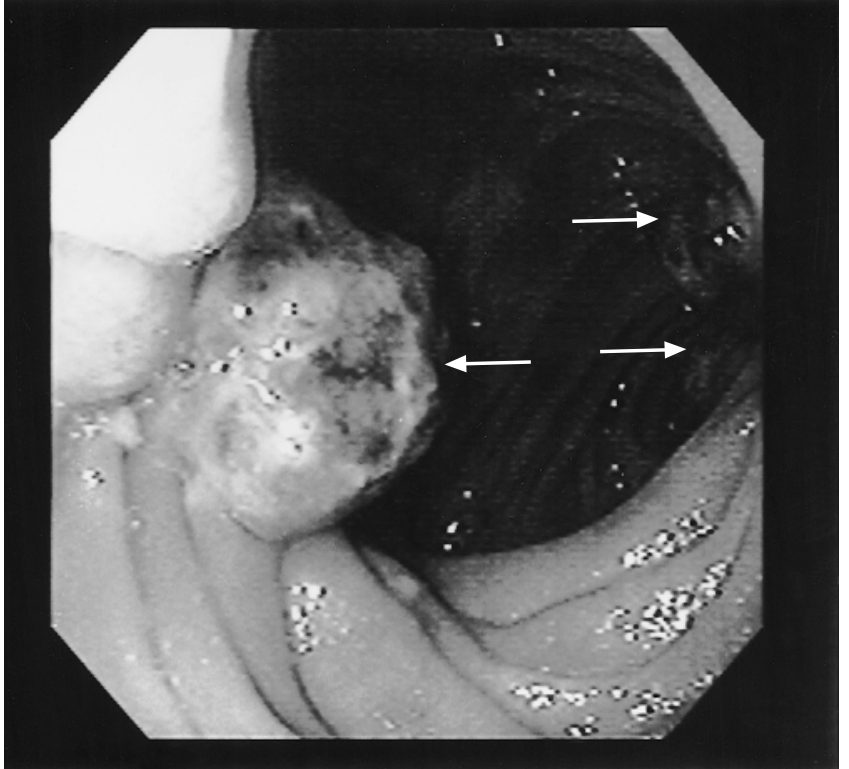

Fig. 5. Endoscopy showed elevated, broad-based tumors in the jejunal pouch along the suture line created by the linear stapler (arrows)

\section{Discussion}

We have reported a case of suture line recurrence in the jejunal pouch after total gastrectomy for advanced gastric cancer. To the best of our knowledge, no intrapouch recurrence of gastric cancer has been reported in the literature (MEDLINE search 1978-1998). In contrast, suture line recurrence has been described after resection for colorectal cancer. Since exfoliation of cancer cells is a well documented phenomenon, the implantation of cancer cells during performance of the anastomosis has been considered a possible cause of suture line recurrence [2-4].

In 1954, McGrew et al. [5] isolated exfoliated colorectal cancer cells in the lumen of resected specimens, but did not determine their viability. In later studies, Rosenberg et al. [6] reported no viable cells in luminal washings, while Umpleby et al. [4] demonstrated viable cells in $70 \%$ of specimens, as assessed by trypan blue exclusion. Fermor et al. [7] demonstrated the proliferative and metastatic potential of exfoliated cancer cells by their ability to grow as xenotransplants in immune-deprived mice. Skipper et al. [8] demonstrated that colonies grew in vitro in monolayer culture derived from luminal mucus specimens. These results indicate that a proportion of exfoliated colorectal cancer cells can undergo proliferation and form metastases, thus supporting their potential role in the etiology of suture line recurrence. Furthermore, occasional reports of carcinomatous deposits occurring on iatrogenic anal fissures suggest that exfoliated cancer cells are poten-

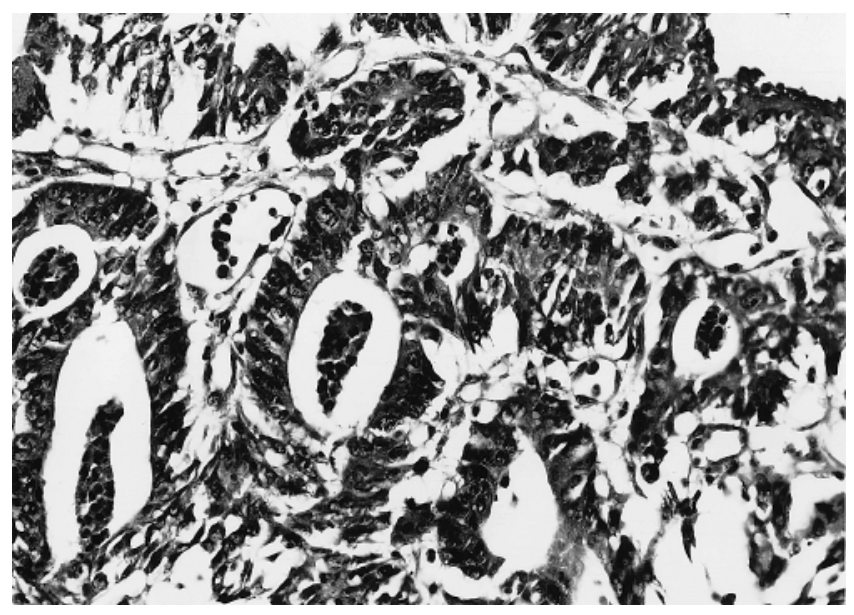

Fig. 6. Well differentiated tubular adenocarcinoma (tub 1) was revealed in the biopsy specimens. $H \& E, \times 80$

tially capable of implantation and proliferation on raw surfaces such as those of surgical anastomoses $[9,10]$.

To prevent implantation by exfoliated cancer cells, irrigation of the intestinal lumen with various cytotoxic agents prior to the creation of the anastomosis has been recommended [11]. The incidence of suture line recurrences was reduced from $10 \%-16 \%$ to $2 \%-$ $3 \%$ following the introduction of measures designed to kill exfoliated cancer cells at operation [7]. Povidone-iodine, at all concentrations, and chlorhexidinecetrimide, at a $10 \%$ concentration or stronger, were recommended as the irrigation agents to achieve neartotal cell kill [11]. Complete lavage of the intestinal lumen with non-cytotoxic agents such as water or saline has also decreased the incidence of suture line recurrence [2].

We propose two causes for the suture line recurrence in our patient: (1) Exfoliated cancer cells in the jejunal mucus may have been implanted at the anastomosis. The jejunal pouch was $30-40 \mathrm{~cm}$ distal to the primary gastric cancer, but it is possible that exfoliated cancer cells may travel such a distance. Umpleby et al. [4] isolated viable cancer cells $35 \mathrm{~cm}$ proximal to a cecal cancer. While irrigation of the jejunal loop with a cytotoxic or non-cytotoxic agent prior to the anastomosis may prevent such implantation, it may be technically difficult to eliminate cancer cells completely. (2) Cancer cells on the stapling device may have been implanted. As we used a linear stapler with a non-disposable knife at the cutting of the duodenal cap, it is possible that the knife was contaminated by the cancer cells. It is therefore recommended to change to a new device or to clear the device with a cytotoxic agent prior to carrying out the reconstruction. It is not apparent how to prevent the implantation, but early diagnosis and salvage surgery should be able to save the patient. 


\section{Conclusion}

Suture line recurrence can occur in gastric cancer operations, with the implantation of exfoliated intraluminal cells constituting a probable mechanism. This rare, but important, phenomenon appears to be preventable.

\section{References}

1. Japanese Gastric Cancer Association. Japanese classification of gastric carcinoma. 2nd English ed. Gastric Cancer 1998;1:10-24.

2. Gertsh P, Baer HU, Kraft R, Madden GJ, Altermatt HJ. Malignant cells are collected on circular staplers. Dis Colon Rectum 1992;35:238-41.

3. Long RTL, Edwards RH. Implantation metastasis as a cause of local recurrence of colorectal carcinoma. Am J Surg 1989;157: 194-201.
4. Umpleby HC, Fermor B, Symes MO, Williamson RCN. Viability of exfoliated colorectal carcinoma cells. Br J Surg 1984;71:659-63.

5. McGrew EA, Laws JF, Cole WH. Free malignant cells in relation to recurrence of carcinoma of the colon. JAMA 1954;154:1251-4.

6. Rosenberg IL, Russel CW, Giles GR. Cell viability studies on the exfoliated colonic cancer cell. Br J Surg 1978;65:188-90.

7. Fermor B, Umpleby HC, Lever JV, Symes MO, Williamson RCN. Proliferative and metastatic potential of exfoliated colorectal cancer cells. J Natl Cancer Inst 1986;76:347-9.

8. Skipper D, Cooper AJ, Marston JE, Taylor I. Exfoliated cells and in vitro growth in colorectal cancer. Br J Surg 1987;74:1049-52.

9. Friend DJD, Corson J, Kramer E, Gallagher P, Prescott R. Cutaneous perianal recurrence of cancer after anterior resection using the EEA stapling device. Ann R Coll Surg Engl 1992;74:142-3.

10. Norgren J, Sevensson JO. Anal implantation metastasis from carcinoma of the sigmoid colon and rectum - a risk when performing anterior resection with the EEA stapler? Br J Surg 1985;72: 602.

11. Umpleby HC, Williamson RCN. The efficacy of agents employed to prevent anastomotic recurrence in colorectal carcinoma. Ann R Coll Surg Engl 1984;66:192-4. 